\title{
Stroke and TIA mimics in patients referred to a neurological emergency department by non-ambulance physicians, ambulance physicians and paramedics
}

\author{
Dorota Kozera-Strzelińska ${ }^{1}$, Michał Karliński ${ }^{1}$, Grzegorz Rak ${ }^{2}$, Magdalena Wojdacz ${ }^{1}$ \\ Halina Sienkiewicz-Jarosz ${ }^{3}$, Iwona Kurkowska-Jastrzębska ${ }^{1}$ \\ ${ }^{1}$ 2nd Department of Neurology, Institute of Psychiatry and Neurology, Warsaw, Poland \\ ${ }^{2}$ 2nd Faculty of Medicine, Medical University of Warsaw, Poland \\ ${ }^{3} 1$ st Department of Neurology, Institute of Psychiatry and Neurology, Warsaw, Poland
}

\section{ABSTRACT}

Introduction. Our aim was to compare the structure and management of conditions mimicking acute cerebrovascular events (ACE) defined as stroke or transient ischaemic attack between patients referred directly to a neurological emergency department (ED) by non-ambulance physicians, ambulance physicians and paramedics.

Methods. This is a retrospective study of 802 consecutive patients referred to a Polish urban neurological ED with a prehospital suspicion of ACE between January and December 2014.

Results. After proper neurological assessment, ACE was excluded in 258 (32.2\%) patients. The ratios of neurological to non-neurological ACE mimics were similar across all groups (35:93 for non-ambulance physicians, 22:39 for ambulance physicians, and 28:39 for paramedics). The most frequent conditions mimicking ACE were vertigo (14.0\%), headache (9.7\%), seizures (7.0\%), blood hypertension (7.0\%), electrolyte and metabolic disturbances (5.4\%), infections (4.7\%) and syncope (4.3\%). There were no major differences between patients with ACE-mimics referred by ambulance physicians and referred by paramedics in terms of demographic, previous medical history, extent of diagnostic workup, final diagnosis or further management (neurological admission in $42.6 \%$ and $28.4 \%$ of cases). However, the characteristics and management of ACE mimics referred by non-ambulance physicians were slightly different, including a lower need for hospital admission (neurological admission in $21.5 \%$ of cases).

Conclusions. There seem to be no major differences in the structure, early diagnostic approach or management of ACE mimics between referrals from ambulance physicians and ambulance paramedics, which provides reassurance to healthcare systems that rely solely on paramedics. Mimics referred by non-ambulance physicians appear different in structure and are less resource-consuming.

Key words: stroke, transient ischaemic attack, misdiagnosis, ambulance, paramedics, emergency department (Neurol Neurochir Pol 2019; 53 (1): 83-89)

\section{Introduction}

Contemporary evidence-based acute stroke treatment is highly oriented around reperfusion therapies, and it aims at constant optimisation of treatment logistics, which particularly refers to shortening door-to-needle or door-to-groin time [1]. Such an approach exerts additional pressure on Emergency Department (ED) personnel and makes

Address for correspondence: Michal Karliński, $2^{\text {nd }}$ Department of Neurology, Institute of Psychiatry and Neurology, Warsaw, Poland e-mail: mkarlinski@ipin.edu.pl 
room for diagnostic mistakes [1]. Not all patients initially suspected of having acute stroke actually suffer from cerebral ischaemia [2-5]. Incorrect diagnoses are made mostly in the prehospital setting but may also happen in the ED, especially if the initially attending physician is not a neurologist or a stroke physician [6]. In addition, clinical signs and symptoms combined with computed tomography (CT) and basic laboratory tests may sometimes be insufficiently conclusive to establish a final diagnosis within the first few hours after presenting to the ED. They sometimes require prolonged observation or magnetic resonance (MR) of the brain, which is not always easily available $24 / 7$ and not always feasible [1]. Previous studies have shown that stroke mimics may account for as much as half of all suspected strokes, with a very wide range (2\% to $47 \%$ ) depending on the setting and methodology [2,7-11]. More importantly, up to $10 \%$ of patients treated with intravenous thrombolysis may actually suffer from a condition other than ischaemic stroke $[2,12]$. Therefore, clinicians should be aware of the most common stroke mimics that need to be addressed in the acute diagnostic workup at the ED. It also seems reasonable to account for country-specific features of emergency healthcare and patients' attitude towards acute illnesses. In Poland, the great majority of patients suspected of stroke within the therapeutic window for thrombolysis or thrombectomy are brought to the hospital by the ambulance with either a physician or a paramedic onboard.

The aim of our study was to compare the structure and management of conditions misdiagnosed in the prehospital setting as acute cerebrovascular events (ACE), defined as stroke or transient ischaemic attack, between patients referred directly to neurological ED by non-ambulance physicians, ambulance physicians and paramedics.

\section{Material and methods}

Our ED provides neurological and stroke care for approximately 350,000 inhabitants of a highly urban area (the southern part of Warsaw and neighbouring Polish towns). The hospital's profile is solely neuropsychiatric, with a neurosurgical ward and interventional neuroradiology. The ED is staffed 24 hours a day and 7 days a week with either a senior neurologist or a neurologist in training. Patients reporting to the ED are referred directly by non-ambulance physicians (mostly general practitioners but also outpatient specialists), ambulance physicians, ambulance paramedics or by themselves without any formal referral.

Brain CT, brain MR or CT-angiography are easily available at the ED and, if medically justified, the imaging can be performed without the need for subsequent admission to the neurological ward.

\section{Study design}

We retrospectively reviewed both paper and electronic source medical documentation of consecutive patients who reported to our neurological ED between 1st January 2014 and 31st December 2014 to identify all cases with a prehospital diagnosis of ACE. Patients who reported without any formal referral were not included in the analysis.

Data were extracted using a predefined form that included information about patient gender, age, type of referring entity, prehospital diagnosis (stroke or transient ischaemic attack or syndrome description highly suggestive of ACE), history of stroke, history of seizures, diagnostic workup undertaken at the ED (brain imaging, blood test), final diagnosis and the decision about admission or further referral.

This paper follows the Strengthening the Reporting of Observational Studies in Epidemiology (STROBE) Statement [13].

\section{Statistical analysis}

Categorical variables were presented as a number of valid observations and proportions calculated with exclusion of unknown values from the denominator. Continuous variables due to non-normal distribution (a Shapiro-Wilk test) were presented as a median with interquartile range (1st quartile to 3rd quartile, Q1-Q3).

Comparisons between patients referred by non-ambulance physicians, ambulance physicians and paramedics were done using chi square test or Kruskal-Wallis test, as appropriate. Pairwise differences were additionally tested only if the overall test for significance was positive $(p<0.05)$. Such an approach allowed us to reduce the risk of type I error without losing power by applying the Bonferroni correction.

$P$ values of $<0.05$ were considered statistically significant. Calculations were carried out using Dell STATISTICA 13.0 software package (Dell, Round Rock, TX, USA).

\section{Results}

During the 12-month study period there were 639 admissions for stroke or TIA in total, including 51 patients who reported to the ED without any referral and 95 patients with a prehospital diagnosis other than ACE. Of 802 patients who were referred to our neurological ED with a suspicion of ACE, 258 (32.2\%) had a final diagnosis other than stroke or TIA. Approximately half of ACE mimics $(n=130)$ was referred by outpatient physicians and the other half by ambulance physicians $(n=61)$ or ambulance paramedics $(n=67)$ (Fig. 1). The ratios of neurological to non-neurological conditions mimicking ACE were similar across all three abovementioned groups (35:93, 22:39 and 28:39, respectively). 


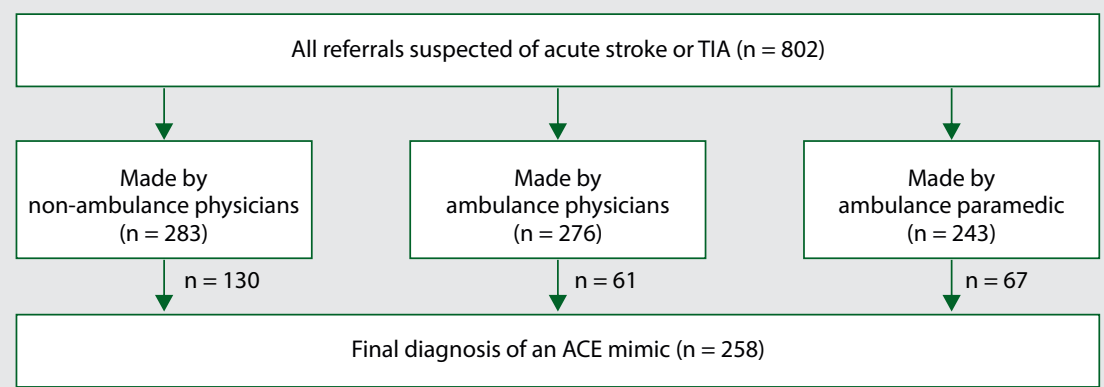

Figure 1. The structure of patients referred directly to neurological ED with a pre-hospital suspicion of ACE according to the type of the referring entity

Table 1. Conditions most frequently mimicking ACE

$\begin{array}{lccc} & \mathbf{n} & \text { Percentage within each group } & \text { Percentage overall (N= 258) } \\ \text { Neurological mimic }(\mathbf{n}=\mathbf{1 7 1}) & 171 & 100 \% & 66.3 \% \\ \text { Vertigo } & 36 & 21.1 \% & 14.0 \% \\ \text { Headache } & 25 & 14.6 \% & 9.7 \% \\ \text { Seizures } & 18 & 10.5 \% & 7.0 \% \\ \text { Brain tumour } & 17 & 9.9 \% & 6.6 \% \\ \text { Sequels of cerebral infarction } & 14 & 8.2 \% & 5.4 \% \\ \text { Bell's palsy } & 13 & 7.6 \% & 5.0 \% \\ \text { Other neurological conditions } & 48 & 28.1 \% & 18.6 \% \\ \text { Non-neurological mimic }(\mathbf{n}=87) & 87 & 100 \% & 33.7 \% \\ \text { Very high blood pressure } & 18 & 20.7 \% & 7.0 \% \\ \text { Metabolic and electrolyte disturbances } & 14 & 16.1 \% & 5.4 \% \\ \text { Infections } & 12 & 13.8 \% & 4.7 \% \\ \text { Syncope } & 11 & 12.6 \% & 4.3 \% \\ \text { Cardiac condition } & 6 & 6.9 \% & 2.3 \% \\ \text { Alcohol abuse } & 5 & 5.7 \% & 1.9 \% \\ \text { Other non-neurological conditions } & 21 & 24.1 \% & 8.1 \%\end{array}$

Patients incorrectly suspected of ACE were in $66.3 \%$ of cases eventually diagnosed with a neurological condition, most frequently vertigo (14.0\%), headache (9.7\%) and seizures (7.0\%). Among non-neurological ACE mimics, the most frequent were blood hypertension (7.0\%), electrolyte and metabolic disturbances (5.4\%), infections (4.7\%) and syncope (4.3\%) (Tab. 1). The category of other neurological ACE mimics included a variety of conditions, mostly traumatic head injuries, side effects of neuroleptics or hypnotics, exacerbations of dementia or parkinsonism and single nerve pathologies. Other non-neurological ACE mimics were predominantly psychosomatic disturbances and exacerbations of chronic pulmonary or cardiac diseases.

There were no major differences between patients with ACE-mimics referred by ambulance physicians and paramedics in terms of demographics, previous medical history, extent of diagnostic workup, final diagnosis and the decision about admission to the neurological ward or direct referral to non-neurological ED in another hospital (Tab. 2).

However, ACE mimicking patients referred by nonambulance physicians suffered significantly more frequently from headache and significantly less frequently from metabolic and electrolyte disturbances than patients referred either by paramedics or by ambulance physicians (Fig. 2). They also tended to have a less frequent history of seizures, less often required blood sample analyses to establish the final diagnosis, and less frequently were eventually admitted to the neurological ward or referred directly to a non-neurological ED in another hospital (Tab. 2). Additionally, compared to the ACE-mimics referred by ambulance physicians, they were 

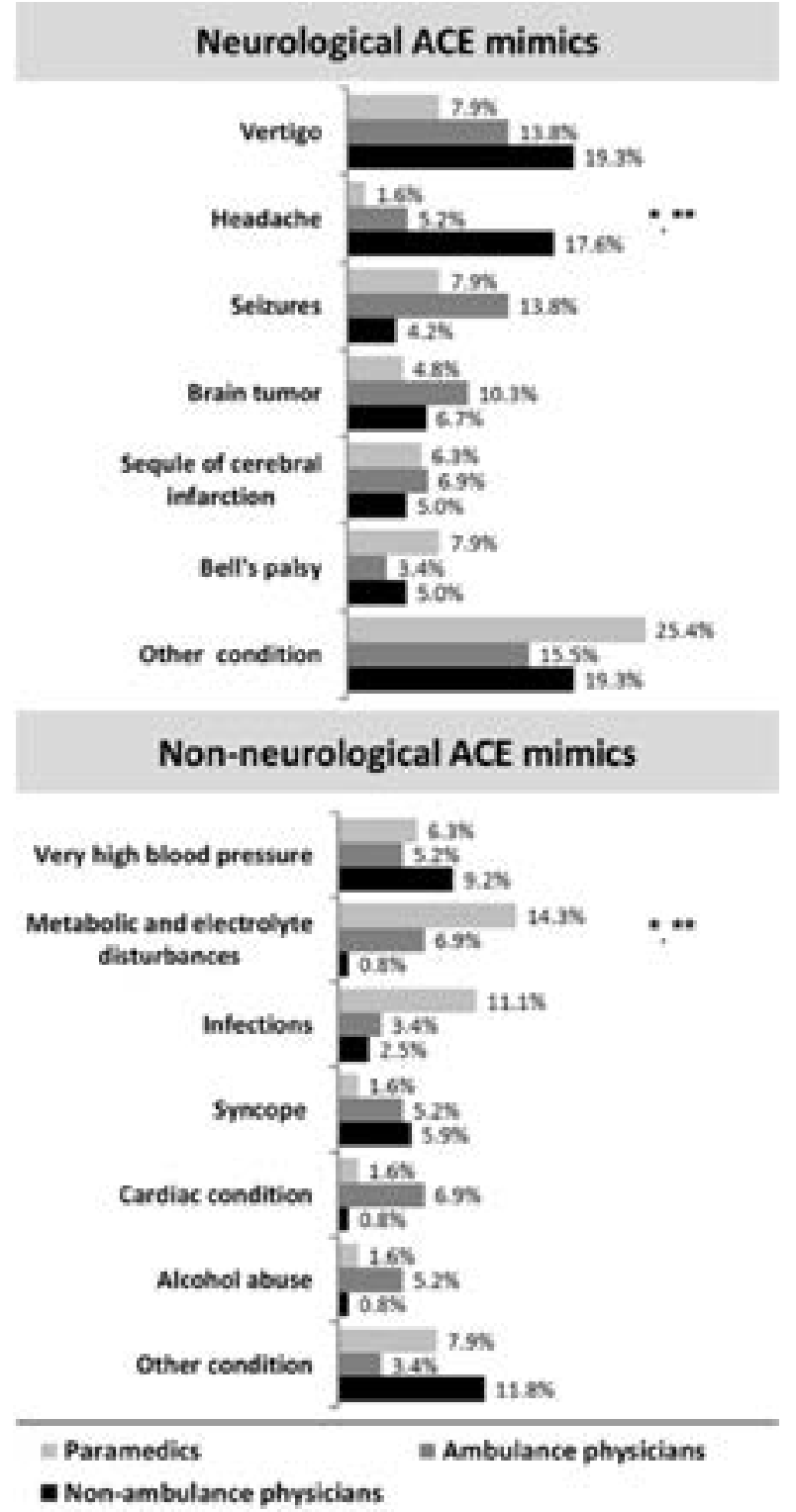

Figure 2. Most frequent ACE mimics according to the type of the referring entity. ${ }^{*}, p<0.05$ for comparison between non-ambulance physicians and ambulance physicians; **, $p<0.05$ for comparison between non-ambulance physicians and ambulance paramedics

more often female, less often had a history of stroke or TIA (transient ischaemic attack), and less often required admission to the neurological ward (Tab. 2). Patients referred by non-ambulance physicians were significantly younger and less often required brain imaging to exclude ACE than the patients referred by paramedics (Tab. 2). In terms of combined direct neurological admissions of ACE mimics or their re-referral to non-neurological EDs, both ambulance physicians and paramedics were superior to non-ambulance physicians (Tab. 2). However, the proportion of solely neurological admissions was significantly higher only among patients with ACE mimics referred by ambulance physicians (Tab. 2).

\section{Discussion}

To the best of our knowledge, this is the first study that has directly evaluated the structure and management of conditions incorrectly suspected in the prehospital setting of being acute stroke or TIA between three major types of referring entities in a healthcare system that employs ambulances with either onboard physicians or paramedics. In Poland, every patient suspected of stroke is immediately transported by emergency services to the nearest hospital with a stroke unit $[1,14]$. Additionally, the Polish healthcare system economically promotes admissions of patients with TIA. According to the American Heart Association, TIA is episodic and transient neurological dysfunctions caused by focal brain, spinal cord, or retinal ischaemia, without acute infarction but with a high short-term risk of subsequent stroke [15]. It is reasonable to admit to the hospital patients who present during the first 72 hours after TIA and have an $\mathrm{ABCD}^{2}$ score $\geq 3$. Hospital admission is also recommended if a diagnostic evaluation cannot be rapidly completed on an outpatient basis [16]. Moreover, in some TIA patients focal symptoms are still present at the time of prehospital evaluation or even initial contact with the ED. Therefore it is reasonable to approach them in the acute phase in a similar manner to patients with suspected stroke, combining those two groups into suspected ACE [6]. As the diagnosis of the ACE is mainly based on clinical symptoms, it may sometimes lead to initiation of intravenous thrombolysis in a non-stroke patient $[1,2,12,17]$. For that reason, some authors propose MR as the first choice imaging modality in hyperacute and acute stages to actually visualise the responsible ischaemic lesion $[8,9,18,19]$. However, MR is not always easily available $24 / 7$, is not always feasible, and is more costly and time-consuming $[1,17,20-22]$. It may also be false negative in lacunar syndromes [23-25]. Therefore, guidelines still promote non-contrast $\mathrm{CT}$ as a simple tool for the exclusion of haemorrhage in otherwise clear cases [1]. In our material none of the ACE mimics received thrombolysis.

Our data shows that patients incorrectly suspected of ACE by non-ambulance physicians were in many respects different from the quite homogenous group of patients referred by the ambulance physicians and ambulance paramedics. Results from previous studies addressing the issue of ACE mimics are not uniform. The most frequent misdiagnoses have included seizures (7-21\%), vertigo (2-5\%), brain tumours (4-15\%), metabolic and electrolyte disturbances (4-24\%), infections $(7-16 \%)$ and functional disorders (1-8\%), which only partially overlap with our results (vertigo followed by metabolic and electrolyte disturbances, headache, seizures and brain tumours without any functional disorders) [10, 11, 26-31].

Such heterogeneity may reflect system- and patient-related differences that limit generalisability and complicate direct comparisons between particular studies. In our previous analysis of patients with ACE mimics referred by physicians in the year 2006/2007, non-ambulance physicians less often 
Table 2. Characteristics of patients with a final diagnosis of ACE mimic according to type of referring entity

\begin{tabular}{|c|c|c|c|c|c|}
\hline & $\begin{array}{c}\text { Non-ambulance } \\
\text { physicians }(n=130)\end{array}$ & $\begin{array}{c}\text { Ambulance } \\
\text { physician }(n=61)\end{array}$ & $\begin{array}{c}\text { Ambulance } \\
\text { paramedic }(n=67)\end{array}$ & Overall P & $\begin{array}{l}\text { Pairwise } \\
\text { differences }\end{array}$ \\
\hline \multicolumn{6}{|l|}{ General information } \\
\hline Male sex, no. [\%] & $39(30.0)$ & $28(45.9)$ & $29(43.3)$ & 0.052 & * \\
\hline Age [years], median [IQR] & $66(54-79)$ & $72(62-79)$ & $73(61-84)$ & 0.015 & $* *$ \\
\hline \multicolumn{6}{|l|}{ Known medical history } \\
\hline Previous stroke or TIA, no. [\%] & $24(18.5)$ & $22(36.1)$ & $17(25.4)$ & 0.030 & * \\
\hline Previous seizures, no. [\%] & $2(1.5)$ & $5(8.2)$ & $5(7.5)$ & 0.056 & $*^{*}, *$ \\
\hline \multicolumn{6}{|l|}{ Prehospital diagnosis, no. [\%] } \\
\hline Stroke & $60(46.2)$ & $35(57.4)$ & $39(58.2)$ & 0.196 & \\
\hline TIA & $58(44.6)$ & $24(39.3)$ & $21(31.3)$ & & \\
\hline Syndrome description & $12(9.2)$ & $2(3.3)$ & $7(10.5)$ & & \\
\hline \multicolumn{6}{|l|}{ Diagnostic workup at Neurological ED } \\
\hline \multicolumn{6}{|l|}{ Brain imaging, no. [\%] } \\
\hline None & $64(49.2)$ & $28(45.9)$ & $21(31.3)$ & 0.074 & \\
\hline Non-contrast CT & $46(35.4)$ & $25(41.0)$ & $38(56.7)$ & & \\
\hline MR (or CT and then MR) & $30(15.4)$ & $8(13.1)$ & $8(11.9)$ & & \\
\hline $\begin{array}{l}\text { Any brain imaging (vs no imaging), } \\
\text { no. [\%] }\end{array}$ & $66(50.8)$ & $33(54.1)$ & $46(68.7)$ & 0.053 & ** \\
\hline Blood sample analysis, no. [\%] & $45(34.6)$ & $45(73.8)$ & $42(62.7)$ & $<0.001$ & $*{ }^{* * *}$ \\
\hline \multicolumn{6}{|l|}{ Discharge from Neurological ED } \\
\hline \multicolumn{6}{|l|}{ Final decision, no. [\%] } \\
\hline Admission to neurological ward & $28(21.5)$ & $26(42.6)$ & $19(28.4)$ & 0.006 & $*{ }^{* * *}$ \\
\hline Referral to another hospital & $24(18.5)$ & $10(16.4)$ & $21(31.3)$ & & \\
\hline Referral to outpatient clinic & $55(42.3)$ & $19(31.2)$ & $14(20.9)$ & & \\
\hline Referral to general practitioner & $22(16.9)$ & $6(9.8)$ & $12(17.9)$ & & \\
\hline $\begin{array}{l}\text { Direct neurological admission } \\
\text { (vs other), no. [\%] }\end{array}$ & $28(21.5)$ & $26(42.6)$ & $19(28.4)$ & 0.011 & * \\
\hline $\begin{array}{l}\text { Admission or hospital referral } \\
\text { (vs other), no. [\%] }\end{array}$ & $52(40.0)$ & $36(59.0)$ & $40(59.7)$ & 0.008 & $*{ }^{*} * *$ \\
\hline
\end{tabular}

${ }^{*}, \mathrm{p}<0.05$ for comparison between non-ambulance physicians and ambulance physicians; ${ }^{*}, \mathrm{p}<0.05$ for comparison between non-ambulance physicians and ambulance paramedics

confused ACE with vertigo (4\%) and headache (2\%) but more often referred patients with metabolic disturbances (8\%) and cardiac conditions (11\%). On the other hand, ambulance physicians less often misdiagnosed brain tumours (5\%) but more often referred metabolic disturbances (15\%). This shift suggests an overall improvement in the prehospital differential diagnosis between ACE and metabolic disturbances. It should be noted that at that time almost all ambulances were staffed with physicians.

Recent data shows that mimics may account for $2 \%$ to $47 \%$ of suspected strokes and up to $60 \%$ of suspected TIAs [2, 7, 9, $11,19,28,29]$. In our cohort, the overall proportion of ACE mimics was $32.2 \%$, which is slightly lower than the $36.8 \%$ observed in our previous study [6]. Interestingly, this positive trend was present among ambulance physicians $(22.1 \%$ now $v$ s $32.4 \%$ previously) but not among non-ambulance physicians (45.9\% now $v s 46.3 \%$ previously) [6].
Prehospital diagnosis of TIA is particularly challenging because symptoms are transient and usually resolve by the time of proper neurological assessment. There are no laboratory tests for TIA so the diagnosis usually depends on the patient's history combined with the experience of the neurologist [30]. Metabolic and electrolyte disturbances or infections may be mistakenly interpreted as ACE, especially in older patients with multiple comorbidities [31].

Our study has several limitations. It is a retrospective analysis of consecutive cases and relies on standard source medical documentation. However, to maximise the chances of obtaining relevant information, both electronic and paper records were searched. Our findings could be biased towards a better performance of prehospital services than in other multi-profile hospitals. Considering ambulance standard operating procedures, we may safely assume that the great majority of patients from our catchment area suspected of 
stroke by the prehospital services were actually brought in to our ED. However, it is possible that some patients with cardiac and other internal medicine conditions may have been referred to nearby multi-proflile hospitals with the vague label of TIA, especially if the ambulance staff was less certain of the diagnosis. For an assessment of external validity, one should note that in our cohort the prehospital diagnosis of TIA accounted for approximately $52 \%$ of ACE mimics and only $26 \%$ of all ACE referrals. One may also speculate that the ambulance dispatchers tend to send out physicians to more severe cases, which are more likely to be genuine strokes.

\section{Conclusion}

The structure of both neurological and non-neurological ACE mimics depend on the type of referring entity. One may assume that the early differential diagnostic approach towards ACE-labelled patients delivered to the ED by ambulance physicians or paramedics should be similar. This is of major importance considering that they are responsible for prehospital identification of candidates for acute stroke reperfusion therapies. It also gives reassurance to those healthcare systems that rely solely on paramedics. The mimics referred by outpatient physicians may require less diagnostic workup in the ED, and are probably least likely to require any type of hospital admission.

\section{Conflict of interest. None.}

Acknowledgement and financial support. None.

\section{References}

1. Powers WJ, Rabinstein AA, Ackerson T, et al. 2018 Guidelines for the early management of patients with acute ischemic stroke: A guideline for healthcare professionals from the American Heart Association/ American Stroke Association. Stroke. 2018; 49: e46-e110.

2. Tsivgoulis G, Zand R, Katsanos AH, et al. Safety of Intravenous Thrombolysis in Stroke Mimics: Prospective 5-Year Study and Comprehensive Meta-Analysis. Stroke. 2015; 46(5): 1281-1287, doi: 10.1161/ strokeaha.115.009012.

3. Nguyen PL, Chang JJ. Stroke Mimics and Acute Stroke Evaluation: Clinical Differentiation and Complications after Intravenous Tissue Plasminogen Activator. J Emerg Med. 2015; 49(2): 244252, doi: 10.1016/j.jemermed.2014.12.072, indexed in Pubmed: 25802155.

4. Mehta S, Vora N, Edgell RC, et al. Stroke mimics under the drip-and-ship paradigm. J Stroke Cerebrovasc Dis. 2014; 23(5): 844-849, doi: 10.1016/j.jstrokecerebrovasdis.2013.07.012, indexed in Pubmed: 23954600.

5. Huff JS. Stroke mimics and chameleons. Emerg Med Clin North Am. 2002; 20(3): 583-595, indexed in Pubmed: 12379962.

6. Karliński M, Gluszkiewicz M, Członkowska A. The accuracy of prehospital diagnosis of acute cerebrovascular accidents: an observational study. Arch Med Sci. 2015; 11(3): 530-535, doi: 10.5114/ aoms.2015.52355, indexed in Pubmed: 26170845.
7. Liberman AL, Prabhakaran S. Stroke Chameleons and Stroke Mimics in the Emergency Department. Curr Neurol Neurosci Rep. 2017; 17(15): 1-11.

8. Förster $\mathrm{A}$, Griebe $\mathrm{M}$, Wolf $\mathrm{ME}$, et al. How to identify stroke mimics in patients eligible for intravenous thrombolysis? J Neurol. 2012; 259(7): 1347-1353, doi: 10.1007/s00415-011-6354-9, indexed in Pubmed: 22231865.

9. Vilela P. Acute stroke differential diagnosis: Stroke mimics. Eur J Radiol. 2017; 96: 133-144, doi: 10.1016/j.ejrad.2017.05.008, indexed in Pubmed: 28551302.

10. Harbison J, Hossain O, Jenkinson D, et al. Diagnostic accuracy of stroke referrals from primary care, emergency room physicians, and ambulance staff using the face arm speech test. Stroke. 2003; 34(1): 71-76, indexed in Pubmed: 12511753.

11. Rizos T, Ringleb PA, Huttner HB, et al. Evolution of stroke diagnosis in the emergency room-a prospective observational study. Cerebrovasc Dis. 2009; 28(5): 448-453, doi: 10.1159/000235989, indexed in Pubmed: 19738373.

12. Kostulas N, Larsson M, Kall TB, et al. Safety of thrombolysis in stroke mimics: an observational cohort study from an urban teaching hospital in Sweden. BMJ Open. 2017; 7(10): e016311, doi: 10.1136/ bmjopen-2017-016311, indexed in Pubmed: 29084788.

13. Elm Ev, Altman D, Egger M, et al. The Strengthening the Reporting of Observational Studies in Epidemiology (STROBE) statement: guidelines for reporting observational studies. The Lancet. 2007; 370(9596): 1453-1457, doi: 10.1016/s0140-6736(07)61602-x.

14. Postępowanie w udarze mózgu - wytyczne grupy ekspertów sekcji chorób naczyniowych polskiego towarzystwa neurologicznego. Neurologia i Neurochirurgia Polska. 2012; 46(1): 16-19.

15. Kernan W, Ovbiagele B, Black H, et al. Guidelines for the Prevention of Stroke in Patients With Stroke and Transient Ischemic Attack. Stroke. 2014; 45(7): 2160-2236, doi: 10.1161/str.0000000000000024.

16. Easton J, Saver J, Albers G, et al. Definition and Evaluation of Transient Ischemic Attack. Stroke. 2009; 40(6): 2276-2293, doi: 10.1161/ strokeaha.108.192218.

17. Guillan M, Alonso-Canovas A, Gonzalez-Valcarcel J, et al. Stroke mimics treated with thrombolysis: further evidence on safety and distinctive clinical features. Cerebrovasc Dis. 2012; 34(2): 115-120, doi: 10.1159/000339676, indexed in Pubmed: 22854315.

18. Girot M, Marc JB, Wiel E, et al. Impact on patient management of the implementation of a magnetic resonance imaging dedicated to neurological emergencies. J Eval Clin Pract. 2017: 1-7.

19. Nadarajan V, Perry RJ, Johnson J, et al. Transient ischaemic attacks: mimics and chameleons. Pract Neurol. 2014; 14(1): $23-$ 31, doi: 10.1136/practneurol-2013-000782, indexed in Pubmed: 24453269.

20. Piliszek A, Witkowski G, Sklinda K, et al. Comprehensive imaging of stroke - Looking for the gold standard. Neurol Neurochir Pol. 2016; 50(4): 241-250, doi: 10.1016/j.pjnns.2016.04.009, indexed in Pubmed: 27375137.

21. Danière $\mathrm{F}$, Edjlali-Goujon $\mathrm{M}$, Mellerio $\mathrm{C}$, et al. MR screening of candidates for thrombolysis: How to identify stroke mimics? J Neuroradiol. 2014; 41(5): 283-295, doi: 10.1016/j.neurad.2014.05.008, indexed in Pubmed: 25451670

22. Khan NI, Chaku S, Goehl C, et al. Novel Algorithm to Help Identify Stroke Mimics. J Stroke Cerebrovasc Dis. 2017: 3-8.

23. Watts J, Wood B, Kelly A, et al. Stroke syndromes associated with DWI-negative MRI include ataxic hemiparesis and isolated internuc- 
lear ophthalmoplegia. Neurol Clin Pract. 2013; 3(3): 186-191, doi: 10.1212/CPJ.0b013e318296f288, indexed in Pubmed: 29473634.

24. Morita S, Suzuki M, lizuka K. False-negative diffusion-weighted MRI in acute cerebellar stroke. Auris Nasus Larynx. 2011; 38(5): 577-582, doi: 10.1016/j.anl.2011.01.017, indexed in Pubmed: 21330074.

25. Oppenheim C, Stanescu R, Dormont D, et al. False-negative diffusion-weighted MR findings in acute ischemic stroke. AJNR Am J Neuroradiol. 2000; 21(8): 1434-1440, indexed in Pubmed: 11003275.

26. Libman RB, Wirkowski E, Alvir J, et al. Conditions that mimic stroke in the emergency department. Implications for acute stroke trials. Arch Neurol. 1995; 52(11): 1119-1122, indexed in Pubmed: 7487564.

27. Hosseininezhad M, Sohrabnejad R. Stroke mimics in patients with clinical signs of stroke. Caspian J Intern Med. 2017; 8(3): 213-216, doi: 10.22088/cjim.8.3.213, indexed in Pubmed: 28932374.
28. Anathhanam S, Hassan A. Mimics and chameleons in stroke. Clin Med (Lond). 2017; 17(2): 156-160, doi: 10.7861/clinmedicine.17-2-156, indexed in Pubmed: 28365629.

29. Uchino K, Massaro L, Hammer M. Transient Ischemic Attack after Tissue Plasminogen Activator: Aborted Stroke or Unnecessary Stroke Therapy? Cerebrovascular Diseases. 2009; 29(1): 57-61.

30. Vargas M, Costa M, Melo TP, et al. Increase in the Admission of Stroke Mimics after Change in Emergency Shifts. Cerebrovascular Diseases. 2017; 44(1-2): 68-74, doi: 10.1159/000475823.

31. Gropen TI, Gokaldas R, Poleshuck R, et al. Factors related to the sensitivity of emergency medical service impression of stroke. Prehosp Emerg Care. 2014; 18(3): 387-392, doi: 10.3109/10903127.2013.864359, indexed in Pubmed: 24460036. 